TRANSACTIONS OF THE

AMERICAN MATHEMATICAL SOCIETY

Volume 364, Number 3, March 2012, Pages 1193-1210

S 0002-9947(2011)05455-3

Article electronically published on October 25, 2011

\title{
INTERSECTIONS OF DILATATES OF CONVEX BODIES
}

\author{
STEFANO CAMPI, RICHARD J. GARDNER, AND PAOLO GRONCHI
}

\begin{abstract}
We initiate a systematic investigation into the nature of the function $\alpha_{K}(L, \rho)$ that gives the volume of the intersection of one convex body $K$ in $\mathbb{R}^{n}$ and a dilatate $\rho L$ of another convex body $L$ in $\mathbb{R}^{n}$, as well as the function $\eta_{K}(L, \rho)$ that gives the $(n-1)$-dimensional Hausdorff measure of the intersection of $K$ and the boundary $\partial(\rho L)$ of $\rho L$. The focus is on the concavity properties of $\alpha_{K}(L, \rho)$. Of particular interest is the case when $K$ and $L$ are symmetric with respect to the origin. In this situation, there is an interesting change in the concavity properties of $\alpha_{K}(L, \rho)$ between dimension 2 and dimensions 3 or higher. When $L$ is the unit ball, an important special case with connections to E. Lutwak's dual Brunn-Minkowski theory, we prove that this change occurs between dimension 2 and dimensions 4 or higher, and conjecture that it occurs between dimension 3 and dimension 4 . We also establish an isoperimetric inequality with equality condition for subsets of equatorial zones in the sphere $S^{2}$, and apply this and the Brunn-Minkowski inequality in the sphere to obtain results related to this conjecture, as well as to the properties of a new type of symmetral of a convex body, which we call the equatorial symmetral.
\end{abstract}

\section{INTRODUCTION}

Suppose that $K$ is a convex body and $B$ is the unit ball in $\mathbb{R}^{n}$, and $\rho \geq 0$ is such that $\partial K \cap \rho S^{n-1} \neq \emptyset$. Let $\alpha_{K}(\rho)=V(K \cap \rho B)$ be the volume of $K \cap \rho B$ and let $\eta_{K}(\rho)=\mathcal{H}^{n-1}\left(K \cap \rho S^{n-1}\right)$ be the area $((n-1)$-dimensional Hausdorff measure $)$ of $K \cap \rho S^{n-1}$. (See Section 2 for other definitions and notation.) This study originates from an observation of Benguria, Levitin, and Parnovski 1, Lemma 5.1 and Remark 5.2] that when $n=2$ and $K$ is $o$-symmetric, $\eta_{K}(\rho)$ is decreasing and hence $\alpha_{K}(\rho)$ is concave, while this is not true in general when $n \geq 3$. (The result for $n=2$ also follows from a more general one of Campi [5, Lemma 1].)

The authors of [1] were motivated by two conjectures concerning the set $N(K)$ of zeros of the Fourier transform $\widehat{1_{K}}$ of the characteristic function of an $o$-symmetric convex body $K$ in $\mathbb{R}^{n}$. They conjecture that the distance from $N(K)$ to the origin is minimized when $K$ is a ball, and is bounded above by $\sqrt{\lambda_{2}(K)}$, where $\lambda_{2}(K)$ is the second Dirichlet eigenvalue of $K$. Their observation mentioned above is applied to prove some weaker versions of these conjectures in the plane.

Received by the editors February 22, 2010.

2010 Mathematics Subject Classification. Primary 52A20, 52A40; Secondary 52A38.

Key words and phrases. Convex body, intersection, dilatate, Brunn-Minkowski inequality, isoperimetric inequality, symmetral, ball, sphere.

The second author was supported in part by the U.S. National Science Foundation Grant DMS-0603307.

(C)2011 American Mathematical Society Reverts to public domain 28 years from publication 
Motivated by this observation and its application, as well as by connections to E. Lutwak's dual Brunn-Minkowski theory (see Section 2 for more details), we initiate a systematic investigation into the properties of the functions $\alpha_{K}(L, \rho)$ and $\eta_{K}(L, \rho)$ (always considered as functions of $\rho$ ) that give the volume $V(K \cap \rho L)$ and area $\mathcal{H}^{n-1}(K \cap \partial(\rho L))$ for the intersection of $K$ and a dilatate $\rho L$ of $L$. (When $L=B$, we write $\alpha_{K}(\rho)$ and $\eta_{K}(\rho)$ instead of $\alpha_{K}(B, \rho)$ and $\eta_{K}(B, \rho)$.) As far as we are aware, such a study is new, somewhat surprising in view of the basic nature of the operations of intersection and dilatation.

A simple application (see Theorem 3.1) of the Brunn-Minkowski inequality yields that for arbitrary convex bodies $K$ and $L$ in $\mathbb{R}^{n}$ with $o \in L$, the function $\alpha_{K}(L, \rho)^{1 / n}$ is concave, and in general the exponent cannot be improved, even when $L=B \subset K$. However, we show in Theorem 3.2 that if in addition $n=2$ and the origin belongs to the so-called kernel of $K$ with respect to $L$, the exponent can be improved and in fact $\alpha_{K}(L, \rho)$ is concave. In particular, this holds when $K$ and $L$ are planar $o$ symmetric convex bodies (see Corollary 3.3). Specializing further by taking $L=B$, we see that Theorem 3.2 yields the observation in [1, Lemma 5.1], but is of course considerably more general. In this generality, it is not possible to approach this result via the function $\eta_{K}(L, \rho)$, and in any case, we construct after Corollary 3.3 planar $o$-symmetric convex polygons $K$ and $L$ such that $\eta_{K}(L, \rho)$ increases rather than decreases. The final result in Section 3, Theorem 3.4, shows that the exponent $1 / n$ in Theorem 3.1 is optimal for $n \geq 3$ even when $K$ and $L$ are $o$-symmetric.

In Section 4, we focus on the special case when $K$ is $o$-symmetric and $L=B$, the situation in [1, and consider the possibility that $\alpha_{K}(\rho)^{1 /(n-1)}$ is concave. From the above we know that this holds when $n=2$. In Theorem 4.5 by making computations when $K$ is an $o$-symmetric slab, we show that in general the exponent $1 /(n-1)$ would be optimal, and moreover when $n \geq 4$, the exponent $1 / n$ is best possible. However, for this slab $K, \alpha_{K}(\rho)^{1 / 2}$ is concave when $n=3$. The extremal nature of the slab led us to make the intriguing Conjecture 4.6 that if $K$ is an $o$-symmetric convex body in $\mathbb{R}^{3}$, then $\alpha_{K}(\rho)^{1 / 2}$ is concave.

Thus we find that for $o$-symmetric convex bodies, the concavity properties of $\alpha_{K}(L, \rho)$ change between 2 and 3 dimensions, and we conjecture that the concavity properties of $\alpha_{K}(\rho)$ change between 3 and 4 dimensions. If the latter is true, this would be a remarkable and surprising fact. Our attempts to shed light on Conjecture 4.6 via the Brunn-Minkowski inequality in the sphere are described in Section [5. The method allows estimates on the derivative $\eta_{K}^{\prime}(\rho)$ and hence $\alpha_{K}^{\prime \prime}(\rho)$, when they exist - see Theorem 5.2 - but so far has not led to a proof of the conjecture. Instead we obtain in Theorem 5.3 an optimal strengthening of the result in [1, Lemma 5.1] and [5, Lemma 1] that when $n=2$ and $K$ is $o$-symmetric, $\eta_{K}(\rho)$ is decreasing.

In Theorem 6.1, we establish an isoperimetric inequality with equality condition for subsets of equatorial zones in the sphere $S^{2}$. This is combined with the method described in the previous paragraph in Theorem 7.1, which states that $\eta_{K}(\rho) / \rho$ is decreasing when $K$ is an o-symmetric convex body in $\mathbb{R}^{3}$. As we explain, this statement is equivalent to the quasiconvexity of a new type of symmetral of $K$, which we call the equatorial symmetral.

In Section 8, we study a related pair of functions. For one of these, defined by $\bar{\alpha}_{K}(L, \rho)=\max _{x \in \mathbb{R}^{n}} V(K \cap(\rho L+x))$, the Brunn-Minkowski inequality is applied again to conclude in Theorem 8.1 that $\bar{\alpha}_{K}(L, \rho)^{1 / n}$ is concave. 
We thank Michael Levitin for drawing our attention to [1], and a referee for a suggestion that shortened our original proof of Theorem 6.1.

\section{Definitions AND PRELiminaries}

As usual, $S^{n-1}$ denotes the unit sphere and $o$ the origin in Euclidean $n$-space $\mathbb{R}^{n}$. The unit ball in $\mathbb{R}^{n}$ will be denoted by $B$. The standard orthonormal basis for $\mathbb{R}^{n}$ will be $\left\{e_{1}, \ldots, e_{n}\right\}$. We write $[x, y]$ for the line segment with endpoints $x$ and $y$.

If $X$ is a set, we denote by $\partial X, \operatorname{int} X$, and conv $X$ the boundary, interior, and convex hull of $X$, respectively.

If $X$ and $Y$ are subsets of $\mathbb{R}^{n}$, their vector or Minkowski sum is

$$
X+Y=\{x+y: x \in X, y \in Y\}
$$

and if $t \in \mathbb{R}$, then

$$
t X=\{t x: x \in X\}
$$

When $t>0, t X$ is called a dilatate of $X$. The set $-X$ is the reflection of $X$ in the origin.

We write $\mathcal{H}^{k}$ for $k$-dimensional Hausdorff measure in $\mathbb{R}^{n}$, where $k=1, \ldots, n$. If $K$ is a $k$-dimensional convex body in $\mathbb{R}^{n}$, then $V(K)$ is its volume $\mathcal{H}^{k}(K)$. The notation $d z$ will always mean $d \mathcal{H}^{k}(z)$ for the appropriate $k=1, \ldots, n$.

By Jensen's inequality for integrals [9, (B.8), p. 414], if $p \leq q$ and a real-valued function $f$ is $q$-concave (that is, $f^{q}$ is concave) on its support, then $f$ is $p$-concave on its support.

We follow Schneider [20] by writing $\kappa_{n}$ for the volume $V(B)$ of the unit ball in $\mathbb{R}^{n}$, so that $\kappa_{n}=\pi^{n / 2} / \Gamma(1+n / 2)$.

A set is o-symmetric if it is centrally symmetric, with center at the origin.

A convex body is a compact convex set with nonempty interior.

Let $K$ and $L$ be convex bodies in $\mathbb{R}^{n}$ with $o \in L$. We write

$$
r(K, L)=\min \{\rho: \partial K \cap \partial(\rho L) \neq \emptyset\}
$$

and

$$
R(K, L)=\max \{\rho: \partial K \cap \partial(\rho L) \neq \emptyset\} .
$$

For $r(K, L) \leq \rho \leq R(K, L)$, we let

$$
\alpha_{K}(L, \rho)=V(K \cap \rho L)
$$

and

$$
\eta_{K}(L, \rho)=\mathcal{H}^{n-1}(K \cap \partial(\rho L)) .
$$

Note that the domain of both functions is $[r(K, L), R(K, L)]$. In the special case when $L=B$, we simplify the notation by writing $r_{K}=r(K, B), R_{K}=R(K, B)$, $\alpha_{K}(\rho)=\alpha_{K}(B, \rho)$, and $\eta_{K}(\rho)=\eta_{K}(B, \rho)$. Note that the domain of the latter functions is $\left[r_{K}, R_{K}\right]$.

It is of course possible to extend these definitions, though we shall only use the previous definitions in our results. For example, when $L=B$, we can define for any bounded Borel set $C$ in $\mathbb{R}^{n}$,

$$
\eta_{C}(\rho)=\mathcal{H}^{n-1}\left(C \cap \rho S^{n-1}\right)=\int_{S^{n-1}} 1_{C}(\rho u) \rho^{n-1} d u,
$$


for all $\rho \geq 0$. Similarly, we can also define $\alpha_{C}(\rho)=\mathcal{H}^{n}(C \cap \rho B)$, for all $\rho \geq 0$. Then we have

$$
\alpha_{C}(\rho)=\int_{\rho B} 1_{C}(x) d x=\int_{0}^{\rho} \int_{S^{n-1}} 1_{C}(r u) r^{n-1} d u d r=\int_{0}^{\rho} \eta_{C}(r) d r .
$$

In 1975, Lutwak 14 initiated the dual Brunn-Minkowski theory (dBMt), in which the intersections of star bodies with subspaces replace the projections of convex bodies onto subspaces in the classical Brunn-Minkowski theory (BMt) expounded in 20. He discovered that integrals over $S^{n-1}$ of products of radial functions (giving the distance from the origin to the boundary) behave like the mixed volumes in the BMt, and he called them dual mixed volumes. Special cases of dual mixed volumes, analogous to the intrinsic volumes in the BMt, are called dual volumes. A formula called the dual Kubota integral recursion (see [15] or [9, Theorem A.7.2]) allows dual volumes to be represented as averages of volumes of intersections with subspaces, just as intrinsic volumes can be represented as averages of projections onto subspaces.

For our purposes, it is useful to define the $i$ th dual volume $\widetilde{V}_{i}(C)$ of any bounded Borel set $C$ in $\mathbb{R}^{n}$, for real $i>0$, by

$$
\widetilde{V}_{i}(C)=\frac{i}{n} \int_{C}\|x\|^{i-n} d x
$$

where integration is with respect to $\mathcal{H}^{n}$. When $C$ is a star body, (3) agrees with the original definition in 14] via a change to polar coordinates; see [11, Theorem 4.1]. Moreover, definition (31) allows dual volumes to be seen as moments of sets, imparting a clear and fundamental geometrical significance to these quantities. For a bounded Borel set $C$ in $\mathbb{R}^{n}$, we have, using (11),

$$
\begin{aligned}
\tilde{V}_{i}(C) & =\frac{i}{n} \int_{C}\|x\|^{i-n} d x=\frac{i}{n} \int_{\mathbb{R}^{n}}\|x\|^{i-n} 1_{C}(x) d x \\
& =\frac{i}{n} \int_{0}^{\infty} \int_{S^{n-1}}\|\rho u\|^{i-n} 1_{C}(\rho u) \rho^{n-1} d u d \rho=\frac{i}{n} \int_{0}^{\infty} \eta_{C}(\rho) \rho^{i-n} d \rho .
\end{aligned}
$$

If $C$ is a star-shaped body with respect to the origin and if $r_{C}$ and $R_{C}$ are the minimum and maximum values of the radial function of $C$, then the previous equation, (2), and integration by parts lead to the formula

$$
\begin{aligned}
\widetilde{V}_{i}(C) & =\frac{i}{n}\left(\rho^{i-n} \alpha_{C}(\rho)||_{0}^{R_{C}}-(i-n) \int_{0}^{R_{C}} \rho^{i-n-1} \alpha_{C}(\rho) d \rho\right) \\
& =\frac{i}{n} \mathcal{H}^{n}(C) R_{C}^{i-n}+\frac{\kappa_{n}(n-i)}{n} r_{C}^{i}+\frac{i(n-i)}{n} \int_{r_{C}}^{R_{C}} \alpha_{C}(\rho) \rho^{i-n-1} d \rho .
\end{aligned}
$$

Thus the functions $\eta_{C}$ and $\alpha_{C}$ are natural components of the dBMt.

In recent years the dBMt has been the subject of quite intense activity. It is now recognized that the dBMt has connections and applications to many areas, such as integral geometry, Minkowski geometry, the local theory of Banach spaces, stereology, and information theory; see [9] and the references given there. Even when restricted to $o$-symmetric convex bodies, the dBMt can count among its successes the solution of the Busemann-Petty problem in [7, 10], 16], 21], and [22. 


\section{Properties of $\alpha_{K}(L, \cdot)$ And $\eta_{K}(L, \cdot)$}

Theorem 3.1. If $K$ and $L$ are convex bodies in $\mathbb{R}^{n}$ with $o \in L$, then $\alpha_{K}(L, \rho)^{1 / n}$ is concave.

Proof. Let $H=K \times[r(K, L), R(K, L)] \subset \mathbb{R}^{n+1}$, let

$$
C=\operatorname{conv}\left\{o,\left\{\left(x_{1}, \ldots, x_{n+1}\right):\left(x_{1}, \ldots, x_{n}\right) \in R(K, L) L, x_{n+1}=R(K, L)\right\}\right\},
$$

and let $M=H \cap C$. Observe that $C$ is a cone and $C \cap\left\{x_{n+1}=\rho\right\}$ is a translation of $\rho L$ along the $x_{n+1}$-axis. Then, identifying the plane $\left\{x_{n+1}=\rho\right\}$ with $\mathbb{R}^{n}$, we have

$$
M \cap\left\{x_{n+1}=\rho\right\}=K \cap \rho L,
$$

for $r(K, L) \leq \rho \leq R(K, L)$, so by the Brunn-Minkowski inequality (see [8], [9, Section B.2], and [20, Section 6.1]) the function

$$
\alpha_{K}(L, \rho)^{1 / n}=V\left(M \cap\left\{x_{n+1}=\rho\right\}\right)^{1 / n}
$$

is concave.

The exponent in the previous result is the best possible, even when $L=B \subset K$. To see this, let $M=B \cap\left\{x_{n} \geq 0\right\}$ be a half-ball containing the origin, so that $r_{M}=0, R_{M}=1$, and $\alpha_{M}(\rho)=\kappa_{n} \rho^{n} / 2,0 \leq \rho \leq 1$. Let $q>1 / n$ and note that $\alpha_{M}(\rho)^{q}=\left(\kappa_{n} / 2\right)^{q} \rho^{n q}$ is not concave. Let $0<s<1 / 2$ and let $K_{s}=M-s e_{n}$. Then $s B \subset K_{s}$ and $\alpha_{K_{s}}$ converges uniformly to $\alpha_{M}$ as $s \rightarrow 0$. Therefore there is an $s_{0}>0$ such that for $s \leq s_{0}, \alpha_{K_{s}}(\rho)^{q}$ is not concave. Letting $K=\left(1 / s_{0}\right) K_{s_{0}}$, we have $B \subset K$ and $\alpha_{K}(\rho)^{q}$ is not concave.

Despite this example, there are situations in which Theorem 3.1 can be strengthened. To state one, let $K$ and $L$ be convex bodies in $\mathbb{R}^{n}$ such that $o \in L$. The relative inradius $\bar{r}(K, L)$ of $K$ with respect to $L$ is defined by

$$
\bar{r}(K, L)=\sup \left\{r: r L+x \subset K \text { for some } x \in \mathbb{R}^{n}\right\} .
$$

The kernel of $K$ with respect to $L$ is denoted by

$$
K_{L}=\{x: \bar{r}(K, L) L+x \subset K\} .
$$

See, for example, [19. Note that if $o \in K_{L}$, then $\bar{r}(K, L)=r(K, L)$ and $r(K, L) L \subset$ $K$ but $s L+x \not \subset K$ for any $s>r(K, L)$ and $x \in \mathbb{R}^{n}$.

Theorem 3.2. If $K$ and $L$ are convex bodies in $\mathbb{R}^{2}$ such that $o \in K_{L}$, then $\alpha_{K}(L, \rho)$ is concave.

Proof. It suffices to show that

$$
\alpha_{K}(L, \rho+h)-\alpha_{K}(L, \rho) \leq \alpha_{K}(L, \rho)-\alpha_{K}(L, \rho-h),
$$

for every $\rho, h>0$ such that $[\rho-h, \rho+h] \subset[r(K, L), R(K, L)]$. If we let

$$
C=(\rho+h) L \backslash \rho L \text { and } D=\rho L \backslash(\rho-h) L,
$$

then we want to show that $\mathcal{H}^{2}(C \cap K) \leq \mathcal{H}^{2}(D \cap K)$. The set int $K \backslash r(K, L) L$ consists of countably many components. Let $E$ be any such component, and let $C_{1}=E \cap \operatorname{int} C$ and $D_{1}=E \cap$ int $D$ be the corresponding (possibly empty) components of int $(C \cap K)$ and int $(D \cap K)$, respectively. Then it suffices to prove that $\mathcal{H}^{2}\left(C_{1}\right) \leq \mathcal{H}^{2}\left(D_{1}\right)$

The component $D_{1}$ is bounded by four arcs, each contained in $\partial K$, or $\partial((\rho-h) L)$, or $\partial(\rho L)$. Let $x_{1}, y_{1}, y_{2}$, and $x_{2}$ be the endpoints of these arcs, labeled clockwise 
and such that $x_{1}$ and $x_{2}$ belong to $(\rho-h) L$. For $i=1,2$, the line through $x_{i}$ and $y_{i}$ intersects $\partial((\rho+h) L)$ in a point $z_{i}$, say.

Let $C_{1}^{\prime} \supset C_{1}$ be the region bounded by $\left[y_{1}, z_{1}\right],\left[y_{2}, z_{2}\right]$, the arc in $\partial(\rho L)$ between $y_{1}$ and $y_{2}$, and the arc in $\partial((\rho+h) L)$ between $z_{1}$ and $z_{2}$. Similarly, let $D_{1}^{\prime} \subset D_{1}$ be the region bounded by the line segments $\left[x_{1}, y_{1}\right]$ and $\left[x_{2}, y_{2}\right]$, the arc in $\partial((\rho-h) L)$ between $x_{1}$ and $x_{2}$, and the arc in $\partial(\rho L)$ between $y_{1}$ and $y_{2}$.

The lines $l_{i}$ containing the segments $\left[x_{i}, y_{i}\right], i=1,2$, are either parallel or meet at a point $w \neq o$. Let $u \in S^{1}$ be parallel to $l_{1}$ and $l_{2}$ in the first case, and let $u=-w /\|w\|$ in the second case. We claim that in the second case, $[o, w] \cap C_{1}^{\prime} \neq \emptyset$. Indeed, if this is not true, then the ray emanating from the origin in the direction $u$ meets $C_{1}^{\prime}$. Note that the closed cone $T$ with apex at $w$ bounded by $l_{1}$ and $l_{2}$ is such that $r(K, L) L \subset K \cap T$. Let $m_{i}, i=1,2$, be the lines parallel to $l_{i}$ and tangent to $r(K, L) L$ at $p_{i}$, say, and let $m_{i}$ meet $\partial(\rho L)$ at $q_{i}$. Then for $i=1,2$, we have $p_{i}, q_{i} \in K$ and the relative interior of the line segment $\left[p_{i}, q_{i}\right]$ is contained in int $K$. From this we see that for sufficiently small $\varepsilon>0$ we have $r(K, L) L+\varepsilon u \subset \operatorname{int} K$ and hence that there is an $r>r(K, L)$ such that $r L+\varepsilon u \subset K$, contradicting the definition of $r(K, L)$ and proving the claim.

In either of the two cases under consideration, let $S$ be the closed strip bounded by the lines through $y_{1}$ and $y_{2}$ parallel to $u$, and let $C_{1}^{\prime \prime}=S \cap C$ and $D_{1}^{\prime \prime}=S \cap D$. Now $C_{1} \subset C_{1}^{\prime} \subset C_{1}^{\prime \prime}$ and $D_{1}^{\prime \prime} \subset D_{1}^{\prime} \subset D_{1}$, so it suffices to show that $\mathcal{H}^{2}\left(C_{1}^{\prime \prime}\right) \leq$ $\mathcal{H}^{2}\left(D_{1}^{\prime \prime}\right)$.

To this end, choose a Cartesian coordinate system with $o$ as the origin and the $y$-axis parallel to $u$, with the positive $y$-axis intersecting $C_{1}^{\prime}$. Suppose that

$$
L=\{(x, y) \mid f(x) \leq y \leq g(x)\},
$$

for suitable functions $f$ and $g$. Then for any $s>0$, we have

$$
s L=\{(x, y) \mid s f(x / s) \leq y \leq s g(x / s)\} .
$$

It follows that if the lines bounding $S$ correspond to $x=a$ and $x=b$, where $a<0<b$, then

$$
\mathcal{H}^{2}\left(C_{1}^{\prime \prime}\right)=\int_{a}^{b}((\rho+h) g(x /(\rho+h))-\rho g(x / \rho)) d x
$$

and

$$
\mathcal{H}^{2}\left(D_{1}^{\prime \prime}\right)=\int_{a}^{b}(\rho g(x / \rho)-(\rho-h) g(x /(\rho-h))) d x .
$$

Thus the desired inequality $\mathcal{H}^{2}\left(C_{1}^{\prime \prime}\right) \leq \mathcal{H}^{2}\left(D_{1}^{\prime \prime}\right)$ would follow immediately from the inequality

$$
2 \rho g\left(\frac{x}{\rho}\right) \geq(\rho-h) g\left(\frac{x}{\rho-h}\right)+(\rho+h) g\left(\frac{x}{\rho+h}\right) .
$$

But the latter is a direct consequence of the concavity of $g$, since with $t=(\rho+$ $h) /(2 \rho)$, so that $0<t<1$, we have

$$
\frac{x}{\rho}=(1-t) \frac{x}{\rho-h}+t \frac{x}{\rho+h} .
$$

Corollary 3.3. If $K$ and $L$ are o-symmetric convex bodies in $\mathbb{R}^{2}$, then $\alpha_{K}(L, \rho)$ is concave. 
In view of Theorem 3.2 and Corollary 3.3 , it is natural to ask whether under the same hypotheses, the function $\eta_{K}(L, \rho)$ is decreasing. This is false, as the following example shows. Let $K=[-2,2]^{2}$ and $L=\operatorname{conv}\{( \pm 2,0),( \pm 1, \pm 1),( \pm 2, \pm 1)\}$, so that $K$ and $L$ are $o$-symmetric polygons in $\mathbb{R}^{2}$. For $1<\rho<2, K \cap \rho L$ is a hexagon and $K \cap \partial(\rho L)$ consists of the line segment $[(-2,2(\rho-1) / 3),(\rho, \rho)]$, the line segment $[(\rho, \rho),(2, \rho)]$, and their reflections in the origin. Therefore

$$
\eta_{K}(L, \rho)=2\left(\frac{\sqrt{10}(\rho+2)}{3}+2-\rho\right),
$$

for $1<\rho<2$, which actually increases with $\rho$.

For $n \geq 3$, Theorem 3.1 implies that under the hypotheses of Theorem 3.2 or Corollary 3.3. $\alpha_{K}(L, \rho)^{1 / n}$ is concave. Our next result shows that in contrast to the case $n=2$, the exponent is optimal.

Theorem 3.4. If $n \geq 3$ and $q>1 / n$, there are o-symmetric convex bodies $K$ and $L$ in $\mathbb{R}^{n}$ such that $\alpha_{K}(L, \rho)^{q}$ is not concave.

Proof. Let $D$ be an $o$-symmetric $(n-1)$-dimensional ball of radius 1 contained in the plane $\left\{x_{n}=0\right\}$ in $\mathbb{R}^{n}$, and let $L=\operatorname{conv}\left\{D, e_{n},-e_{n}\right\}$, so that $L$ is a double cone. Let $0<r<R$ and let $K=\left\{-r \leq x_{n} \leq r\right\} \cap R L$. Then $r(K, L)=r$ and $R(K, L)=R$. We claim that for $n \geq 3$ and $q>1 / n, \alpha_{K}(L, \rho)^{q}$ is not concave. Indeed, for $\rho \geq r$ we have

$$
\alpha_{K}(L, \rho)=\frac{2 \kappa_{n-1}}{n} \int_{0}^{r}(\rho-t)^{n-1} d t=\frac{2 \kappa_{n-1}}{n}\left(\rho^{n}-(\rho-r)^{n}\right) .
$$

Let $g(\rho)=\alpha_{K}(L, \rho)^{q}$. Then $g^{\prime \prime}(\rho)=q \alpha_{K}(L, \rho)^{q-2} I(\rho)$, where

$$
I(\rho)=(q-1) \alpha_{K}^{\prime}(L, \rho)^{2}+\alpha_{K}(L, \rho) \alpha_{K}^{\prime \prime}(L, \rho) .
$$

Let $r=1$. A direct calculation with $q=1 / n+c$ and $c>0$ shows that

$$
\lim _{\rho \rightarrow 1+} I(\rho)=4 \kappa_{n-1}^{2}\left(c-\frac{n-1}{n}\right)+4 \kappa_{n-1}^{2} \frac{n-1}{n}=4 c \kappa_{n-1}^{2}>0
$$

for all $n \geq 3$. It follows that $g^{\prime \prime}(\rho)>0$ for $\rho$ close to 1 , proving the claim.

\section{Properties of $\alpha_{K}$ AND $\eta_{K}$}

The next result is just the special case $L=B$ of Theorem 3.1 .

Corollary 4.1. If $K$ is a convex body in $\mathbb{R}^{n}$, then $\alpha_{K}(\rho)^{1 / n}$ is concave.

We now establish differentiability properties, some of which we require later. Note firstly that while $\alpha_{K}$ is clearly continuous (meaning, as always, on its domain $\left.\left[r_{K}, R_{K}\right]\right), \eta_{K}$ may not be. For example, let $K \subset \mathbb{R}^{2}$ be the convex hull of $S^{1}$, the $\operatorname{arc}\{(r, \theta): r=2, \pi / 4 \leq \theta \leq \pi / 2\}$ in $2 S^{1}$, and the point $(2 \sqrt{2}, 0)$. Then $\eta_{K}$ has jump discontinuities at $\rho=1$ and $\rho=2$. Observe that this example is easily modified to produce convex bodies with arbitrarily smooth boundaries for which $\eta_{K}$ is not even continuous.

Theorem 4.2. Let $K$ be a convex body in $\mathbb{R}^{n}$.

(i) The left and right one-sided derivatives $\left(\alpha_{K}\right)_{-}^{\prime}$ and $\left(\alpha_{K}\right)_{+}^{\prime}$ of $\alpha_{K}$ exist and $\left(\alpha_{K}\right)_{+}^{\prime} \leq\left(\alpha_{K}\right)_{-}^{\prime}$. We have $\left(\alpha_{K}\right)_{-}^{\prime}=\eta_{K}$ and $\eta_{K}$ is continuous on the left and its one-sided limits exist. There is a countable subset $M$ of $\left[r_{K}, R_{K}\right]$ such that $\alpha_{K}(\rho)$ is differentiable and $\alpha_{K}^{\prime}(\rho)=\eta_{K}(\rho)$ for $\rho \in\left[r_{K}, R_{K}\right] \backslash M$. Consequently, 
the only discontinuities of $\eta_{K}$ are jump discontinuities at points in $M$, and at such a discontinuity we have

$$
\lim _{s \rightarrow \rho+} \eta_{K}(s)<\eta_{K}(\rho) .
$$

Hence $\eta_{K}$ is upper semicontinuous.

(ii) There is a set $N$ of measure zero such that $\alpha_{K}(\rho)$ is twice differentiable, and hence $\eta_{K}(\rho)$ is differentiable for $\rho \in\left[r_{K}, R_{K}\right] \backslash(M \cup N)$.

Proof. For (i), we use Corollary 4.1 and a standard result on concave or convex functions of one variable (see, for example, the statement and proof of [12, Theorem 1.4]) to conclude that if $g_{K}=\alpha_{K}^{1 / n}$, then $\left(g_{K}\right)_{-}^{\prime}$ and $\left(g_{K}\right)_{+}^{\prime}$ exist and are continuous on the left and right, respectively, that $\left(g_{K}\right)_{+}^{\prime} \leq\left(g_{K}\right)_{-}^{\prime}$, and that there is a countable set $M$ of $\left[r_{K}, R_{K}\right]$ such that $g_{K}(\rho)$ is differentiable for $\rho \in\left[r_{K}, R_{K}\right] \backslash M$. Since $\alpha_{K}$ is increasing, the same is true when $g_{K}$ is replaced by $\alpha_{K}$. Now using the convexity of $K$, we see that

$$
\eta_{K}(\rho)=\lim _{s \rightarrow \rho-} \frac{\alpha_{K}(\rho)-\alpha_{K}(s)}{\rho-s}=\left(\alpha_{K}\right)_{-}^{\prime}(\rho) .
$$

This proves (i).

To prove (ii), we similarly apply Corollary 4.1 and another result on concave or convex functions of one variable (see [12, Theorem 1.7]; this is the one-dimensional case of Aleksandrov's theorem on the almost-everywhere twice-differentiability of concave or convex functions, which according to Schneider [20, p. 32] was first observed by Jessen).

As is explained in [12, p. 10], the previous result means that $\eta_{K}^{\prime}(\rho)$ exists almost everywhere in $\left[r_{K}, R_{K}\right]$ in the sense that

$$
\lim _{s \rightarrow \rho} \frac{\eta_{K}(\rho)-\eta_{K}(s)}{\rho-s}
$$

exists for $\rho \in\left[r_{K}, R_{K}\right] \backslash(M \cup N)$, where in the limit $s \in\left[r_{K}, R_{K}\right] \backslash M$.

Theorem 4.3. Let $K$ be a convex body in $\mathbb{R}^{n}$ and let $\varepsilon>0$. There is a convex body $L$ in $\mathbb{R}^{n}$ such that $r_{L}=r_{K}, R_{L}=R_{K}$, the Hausdorff distance between $K$ and $L$ is less than $\varepsilon$, and $\eta_{L}$ is continuous. Moreover, if $K$ is o-symmetric, there is an o-symmetric $L$ with these properties.

Proof. If $\eta_{K}$ is not continuous, then by Theorem 4.2 (i), it has a jump discontinuity, which can only occur if for some $r_{K} \leq \rho<R_{K}$, we have $\mathcal{H}^{n-1}\left(\partial K \cap \rho S^{n-1}\right)>0$. Without loss of generality, we may suppose that $K \subset\left\{-s \leq x_{n} \leq r_{K}\right\}$, where $s \geq r_{K}$ and $s=r_{K}$ if $K$ is $o$-symmetric.

Let $E$ be a nonspherical $o$-symmetric ellipsoid and choose $a>0$ sufficiently small so that if $L^{\prime}=K+a E$, the Hausdorff distance between $K$ and $L^{\prime}$ is less than $\varepsilon$. Using the fact that the support functions of $K, L^{\prime}$, and $E$ are related by $h_{L^{\prime}}=h_{K}+a h_{E}$, it is easy to see that $\mathcal{H}^{n-1}\left(\partial L^{\prime} \cap \rho S^{n-1}\right)=0$ for each $\rho$ and hence that $\eta_{L^{\prime}}$ is continuous. Let $L=L^{\prime} \cap\left\{-s \leq x_{n} \leq r_{K}\right\} \cap R_{K} B$. Then $L$ retains all the desired properties and is $o$-symmetric if $K$ is.

The following result is proved by a straightforward direct argument in [1, Lemma 5.1].

Proposition 4.4. If $K$ is an o-symmetric convex body in $\mathbb{R}^{2}$, then $\eta_{K}(\rho)$ is decreasing and hence $\alpha_{K}(\rho)$ is concave. 
The first statement in Proposition 4.4 is also implied by [5, Lemma 1], which, when $K$ is $o$-symmetric, states that $\rho \sin \left(\eta_{K}(\rho) /(4 \rho)\right)$ is decreasing. Indeed, if $\rho_{1}<\rho_{2}$, then

$$
\rho_{2} \sin \left(\frac{\eta_{K}\left(\rho_{2}\right)}{4 \rho_{2}}\right) \leq \rho_{1} \sin \left(\frac{\eta_{K}\left(\rho_{1}\right)}{4 \rho_{1}}\right) \leq \rho_{2} \sin \left(\frac{\eta_{K}\left(\rho_{1}\right)}{4 \rho_{2}}\right),
$$

and $\eta_{K}\left(\rho_{2}\right) \leq \eta_{K}\left(\rho_{1}\right)$ follows directly. A still stronger result is given in Theorem5.3 below.

That the second statement in Proposition 4.4 follows from the first is a consequence of (2). It also follows immediately from Corollary 3.3. However, as we have seen, the fact that $\eta_{K}(L, \rho)$ is not always decreasing means that the first statement cannot be obtained from a more general result.

Theorem 4.5. If $n=2$ or 3 and $q>1 /(n-1)$, or if $n \geq 4$ and $q>1 / n$, there is an o-symmetric convex body $K$ in $\mathbb{R}^{n}$ such that $\alpha_{K}(\rho)^{q}$ is not concave.

Proof. Let $n \geq 2$, let $R>1$, and let $K=\left\{-1 \leq x_{n} \leq 1\right\} \cap R B$. Then $r_{K}=1$, $R_{K}=R$, and

$$
\alpha_{K}(\rho)=2 \kappa_{n-1} \int_{0}^{1}\left(\rho^{2}-t^{2}\right)^{(n-1) / 2} d t
$$

for $1 \leq \rho \leq R$. As in the proof of Theorem 3.4, we let $g(\rho)=\alpha_{K}(\rho)^{q}$, so that $g^{\prime \prime}(\rho)=q \alpha_{K}(\rho)^{q-2} I(\rho)$, where

$$
I(\rho)=(q-1) \alpha_{K}^{\prime}(\rho)^{2}+\alpha_{K}(\rho) \alpha_{K}^{\prime \prime}(\rho) .
$$

It suffices in each case to show that $I(\rho)$ may be positive.

Suppose that $n=2$. Then

$$
\alpha_{K}(\rho)=2\left(\rho^{2} \arcsin (1 / \rho)+\sqrt{\rho^{2}-1}\right) .
$$

Therefore

$$
I(\rho)=8\left((2 q-1) \rho^{2} \arcsin ^{2}(1 / \rho)-\frac{\arcsin (1 / \rho)}{\sqrt{\rho^{2}-1}}-1\right) .
$$

Since $I(\rho) \rightarrow 16(q-1)$ as $\rho \rightarrow \infty$, we have $I(\rho)>0$ when $q>1$ and $\rho$ (and hence $R)$ is sufficiently large.

If $n=3$, then $\alpha_{K}(\rho)=2 \pi\left(\rho^{2}-1 / 3\right)$. Therefore

$$
I(\rho)=8 \pi^{2}\left((2 q-1) \rho^{2}-1 / 3\right),
$$

so again, we have $I(\rho)>0$ when $q>1 / 2$ and $\rho$ (and hence $R$ ) is sufficiently large.

Now suppose that $n \geq 4$. In view of (44), we have

$$
\lim _{\rho \rightarrow 1+} \alpha_{K}^{\prime}(\rho)=\lim _{\rho \rightarrow 1+} 2 \kappa_{n-1}(n-1) \rho \int_{0}^{1}\left(\rho^{2}-t^{2}\right)^{\frac{n-3}{2}} d t=\kappa_{n-1}(n-1) \frac{\pi^{1 / 2} \Gamma\left(\frac{n-1}{2}\right)}{\Gamma\left(\frac{n}{2}\right)},
$$$$
\lim _{\rho \rightarrow 1+} \alpha_{K}(\rho)=\kappa_{n}=\kappa_{n-1} \frac{\pi^{1 / 2} \Gamma\left(\frac{n+1}{2}\right)}{\Gamma\left(\frac{n+2}{2}\right)},
$$ 
and, using the relation $x \Gamma(x)=\Gamma(x+1)$,

$$
\begin{aligned}
\lim _{\rho \rightarrow 1+} \alpha_{K}^{\prime \prime}(\rho)= & \lim _{\rho \rightarrow 1+} 2 \kappa_{n-1}(n-1)(n-3) \rho^{2} \int_{0}^{1}\left(\rho^{2}-t^{2}\right)^{(n-5) / 2} d t \\
& +2 \kappa_{n-1}(n-1) \int_{0}^{1}\left(\rho^{2}-t^{2}\right)^{(n-3) / 2} d t \\
= & \kappa_{n-1}(n-1)(n-3) \frac{\pi^{1 / 2} \Gamma\left(\frac{n-3}{2}\right)}{\Gamma\left(\frac{n-2}{2}\right)}+\kappa_{n-1}(n-1) \frac{\pi^{1 / 2} \Gamma\left(\frac{n-1}{2}\right)}{\Gamma\left(\frac{n}{2}\right)} \\
= & \pi^{1 / 2} \kappa_{n-1}(n-1)^{2} \frac{\Gamma\left(\frac{n-1}{2}\right)}{\Gamma\left(\frac{n}{2}\right)} .
\end{aligned}
$$

Therefore (6), (7), and (8) yield

$$
\begin{aligned}
\lim _{\rho \rightarrow 1+} I(\rho) & =\pi(n-1)^{2} \kappa_{n-1}^{2} \frac{\Gamma\left(\frac{n-1}{2}\right)}{\Gamma\left(\frac{n}{2}\right)}\left((q-1) \frac{\Gamma\left(\frac{n-1}{2}\right)}{\Gamma\left(\frac{n}{2}\right)}+\frac{\Gamma\left(\frac{n+1}{2}\right)}{\Gamma\left(\frac{n+2}{2}\right)}\right) \\
& =\pi\left((n-1) \kappa_{n-1} \frac{\Gamma\left(\frac{n-1}{2}\right)}{\Gamma\left(\frac{n}{2}\right)}\right)^{2}(q-1 / n) .
\end{aligned}
$$

It follows that if $q>1 / n$, then $I(\rho)>0$ when $\rho$ is sufficiently close to 1 . This completes the proof.

Our results so far leave open the following possibility.

Conjecture 4.6. If $K$ is an o-symmetric convex body in $\mathbb{R}^{3}$, then $\alpha_{K}(\rho)^{1 / 2}$ is concave.

Note that when $n=3$, it follows from (5) with $q=1 / 2$ that $\alpha_{K}(\rho)^{1 / 2}$ is indeed concave for the convex body $K$ in Theorem 4.5.

\section{Consequences of the Brunn-Minkowski inequality in the SPhere}

For any set $D$ in $S^{n-1}$, let $D_{\varepsilon}=\left\{x \in S^{n-1}: d(x, D) \leq \varepsilon\right\}$, where $d$ denotes the angular metric in $S^{n-1}$ (i.e., the induced metric in $S^{n-1}$; see [4, p. 10]). The following result is sometimes called the Brunn-Minkowski inequality in the sphere; see [8, p. 380] and the references given there, particularly [4, p. 77].

Proposition 5.1. Let $A$ be an $\mathcal{H}^{n-1}$-measurable subset of $S^{n-1}$ and let $\varepsilon>0$. Let $C$ be a spherical cap in $S^{n-1}$ such that $\mathcal{H}^{n-1}(C)=\mathcal{H}^{n-1}(A)$. Then

$$
\mathcal{H}^{n-1}\left(A_{\varepsilon}\right) \geq \mathcal{H}^{n-1}\left(C_{\varepsilon}\right) \text {. }
$$

Henceforth we denote by $m(s)$ the $\mathcal{H}^{n-1}$-measure of a cap in $S^{n-1}$ with angular radius $s$.

Theorem 5.2. If $K$ is a convex body containing the origin in $\mathbb{R}^{n}$, then

$$
F_{n, K}(\rho)=m^{-1}\left(\eta_{K}(\rho) / \rho^{n-1}\right)+\arccos \left(r_{K} / \rho\right)
$$

is decreasing.

Proof. For $\rho>0$, let

$$
f_{K}(\rho)=\mathcal{H}^{n-1}\left((1 / \rho) K \cap S^{n-1}\right)=\eta_{K}(\rho) / \rho^{n-1}
$$

be the normalized version of $\eta_{K}$. 
Fix $\rho>r_{K}$ and let $0<\varepsilon<\rho-r_{K}$. Let $x \in K \cap \rho S^{n-1}$, let $D_{x}=\operatorname{conv}\left\{r_{K} B, x\right\}$, and note that by convexity,

$$
(1 /(\rho-\varepsilon)) K \cap S^{n-1} \supset(1 /(\rho-\varepsilon)) D_{x} \cap S^{n-1},
$$

a closed cap in $S^{n-1}$. By elementary geometry, we find that the angular radius of this cap is

$$
\phi=\arccos \left(r_{K} / \rho\right)-\arccos \left(r_{K} /(\rho-\varepsilon)\right) .
$$

Let $A=(1 / \rho) K \cap S^{n-1}$. Since (10) holds for any $x \in K \cap \rho S^{n-1}$, we obtain

$$
A_{\phi}=\left((1 / \rho) K \cap S^{n-1}\right)_{\phi} \subset(1 /(\rho-\varepsilon)) K \cap S^{n-1} .
$$

This and Proposition 5.1 yield

$$
f_{K}(\rho-\varepsilon)=\mathcal{H}^{n-1}\left((1 /(\rho-\varepsilon)) K \cap S^{n-1}\right) \geq \mathcal{H}^{n-1}\left(A_{\phi}\right) \geq \mathcal{H}^{n-1}\left(C_{\phi}\right),
$$

where $C$ is a cap in $S^{n-1}$ such that $\mathcal{H}^{n-1}(C)=\mathcal{H}^{n-1}(A)=f_{K}(\rho)$. Choose $\psi$ such that $m(\psi)=f_{K}(\rho)$, i.e. $\psi=m^{-1}\left(f_{K}(\rho)\right)$. Then the angular radius of $C$ is $\psi$, so the angular radius of $C_{\phi}$ is $\psi+\phi$. Therefore (12) implies that

$$
f_{K}(\rho-\varepsilon) \geq m(\psi+\phi)=m\left(m^{-1}\left(f_{K}(\rho)\right)+\phi\right) .
$$

Using (11), we obtain

$$
m^{-1}\left(f_{K}(\rho-\varepsilon)\right)+\arccos \left(r_{K} /(\rho-\varepsilon)\right) \geq m^{-1}\left(f_{K}(\rho)\right)+\arccos \left(r_{K} / \rho\right),
$$

and the theorem follows immediately.

The previous result cannot be improved, in the sense that there are convex bodies $K$ containing the origin in $\mathbb{R}^{n}$ for which the function $F_{n, K}$ is constant. This is the case whenever $K=\operatorname{conv}\{A, r B\}$, where $r=r_{K}>0$ and $A$ is a (possibly degenerate) spherical cap in $R S^{n-1}=R_{K} S^{n-1}$ with angular radius in the interval $[0, \arcsin (r / R)]$.

We can use the argument of Theorem 5.2 to obtain a stronger result than Proposition 4.4. Indeed, it is not difficult to prove that it is also stronger than the result for planar o-symmetric convex bodies from [5, Lemma 1] that was mentioned after Proposition 4.4.

Theorem 5.3. If $K$ is an o-symmetric convex body in $\mathbb{R}^{2}$, then

$$
G_{2, K}=\eta_{K}(\rho) /(4 \rho)+\arccos \left(r_{K} / \rho\right)
$$

is decreasing, and this implies that $\eta_{K}(\rho)$ is decreasing.

Proof. When $K$ is $o$-symmetric in $\mathbb{R}^{2}$, we can use the symmetry and the fact that if $r_{K}<\rho<R_{K}$ and $E$ is a component of $K \cap \rho S^{1}$, then $-E$ is another such component disjoint from $E$ to obtain

$$
f_{K}(\rho-\varepsilon) \geq 2 m\left(m^{-1}\left(f_{K}(\rho) / 2\right)+\phi\right)
$$

instead of (13) in the proof of Theorem 5.2. With this and the fact that $m(s)=2 s$ when $n=2$, it follows as in the proof of Theorem 5.2 that $G_{2, K}$ is decreasing.

Suppose that $\eta_{K}$ is not decreasing, so that there exist $\rho_{1}<\rho_{2}$ such that $\eta_{K}\left(\rho_{1}\right)<$ $\eta_{K}\left(\rho_{2}\right)$. Let $M=\sup \left\{\eta_{K}(\rho): \rho_{1} \leq \rho \leq \rho_{2}\right\}$. By Theorem 4.2(i), $\eta_{K}$ is continuous on the left and upper semicontinuous, so $M$ is a maximum and the set $\left\{s \in\left[\rho_{1}, \rho_{2}\right]\right.$ : $\left.\eta_{K}(s)=M\right\}$ is closed. Let $\rho_{0}$ be the minimum number in this set. Then for $0<\varepsilon \leq \varepsilon_{0}=\rho_{0}-\rho_{1}$, we have

$$
\eta_{K}\left(\rho_{0}-\varepsilon\right)<\eta_{K}\left(\rho_{0}\right)
$$


Next, we observe that the function $g(\rho)=\arccos \left(r_{K} / \rho\right)$ is strictly increasing and concave in $\rho$, so for $0<\varepsilon \leq \varepsilon_{0}$,

$$
\arccos \frac{r_{K}}{\rho_{0}}-\arccos \frac{r_{K}}{\rho_{0}-\varepsilon}=g\left(\rho_{0}\right)-g\left(\rho_{0}-\varepsilon\right)>\varepsilon g^{\prime}\left(\rho_{0}\right)=\frac{\varepsilon r_{K}}{\rho_{0} \sqrt{\rho_{0}^{2}-r_{K}^{2}}} .
$$

Using the fact that $G_{2, K}$ is decreasing, (16), and (15), we obtain

$$
\begin{aligned}
\frac{\eta_{K}\left(\rho_{0}-\varepsilon\right)}{4\left(\rho_{0}-\varepsilon\right)}+\arccos \frac{r_{K}}{\rho_{0}-\varepsilon} & >\frac{\eta_{K}\left(\rho_{0}\right)}{4 \rho_{0}}+\arccos \frac{r_{K}}{\rho_{0}} \\
& >\frac{\eta_{K}\left(\rho_{0}-\varepsilon\right)}{4 \rho_{0}}+\arccos \frac{r_{K}}{\rho_{0}-\varepsilon}+\frac{\varepsilon r_{K}}{\rho_{0} \sqrt{\rho_{0}^{2}-r_{K}^{2}}},
\end{aligned}
$$

and hence

$$
\eta_{K}\left(\rho_{0}-\varepsilon\right)>\frac{4\left(\rho_{0}-\varepsilon\right) r_{K}}{\sqrt{\rho_{0}^{2}-r_{K}^{2}}} .
$$

Using (15), letting $\varepsilon \rightarrow 0$, and letting $\theta_{0}=\arcsin \left(r_{K} / \rho_{0}\right)$, we have $0<\theta_{0}<\pi / 2$ and

$$
\eta_{K}\left(\rho_{0}\right) \geq 4 \rho_{0} \tan \theta_{0}>4 \rho_{0} \theta_{0} .
$$

However, since $K$ is $o$-symmetric, it is contained in a closed $o$-symmetric strip $S$ of width $2 r_{K}$. Therefore

$$
\eta_{K}\left(\rho_{0}\right) \leq V_{1}\left(S \cap \rho_{0} S^{1}\right)=4 \arcsin \left(r_{K} / \rho_{0}\right)=4 \rho_{0} \theta_{0} .
$$

This contradiction proves that $\eta_{K}$ is decreasing.

The previous theorem cannot be improved, since when $K=\left\{-r_{K} \leq x_{2} \leq\right.$ $\left.r_{K}\right\} \cap R B$ where $R>r_{K}$, the function $G_{2, K}$ is constant.

\section{AN ISOPERIMETRIC INEQUALITY FOR EQUATORIAL ZONES IN $S^{2}$}

By a sector of $S^{2}$ we mean the closed region between two lines of longitude, i.e. $\left\{(1, \theta, \phi): \theta_{1} \leq \theta \leq \theta_{2}\right\}$, where $\theta$ is the horizontal angle and $\phi$ the vertical angle in spherical polar coordinates. If $\theta_{2}-\theta_{1}=\gamma$, we denote such a sector by $S(\gamma)$; i.e., this is any sector of $S^{2}$ subtending a horizontal angle of $\gamma$ at the origin.

An equatorial zone in $S^{2}$ is the intersection of $S^{2}$ with a closed slab of the form $\left\{\left(x_{1}, x_{2}, x_{3}\right) \in \mathbb{R}^{3}:-a \leq x_{3} \leq a, 0<a \leq 1\right\}$. We denote this equatorial zone by $E(a)$.

If $A$ is an $\mathcal{H}^{2}$-measurable set in $S^{2}$, we denote by $P(A)$ its perimeter. We follow Burago and Zalgaller [4, p. 106] in defining

$$
P(A)=\inf \left\{\liminf _{i \rightarrow \infty} \mathcal{H}^{1}\left(\partial M_{i}\right)\right\}
$$

where the infimum is taken over all sequences of spherical polygons $M_{i}$ in $S^{2}$ with boundaries $\partial M_{i}$ such that $M_{i} \rightarrow A$ as $i \rightarrow \infty$ in the symmetric difference metric. (Note that the latter condition implies in particular that $\mathcal{H}^{2}\left(M_{i}\right) \rightarrow \mathcal{H}^{2}(A)$ as $i \rightarrow \infty$.) We also follow Burago and Zalgaller [4, p. 69] in defining

$$
\mu_{+}(A)=\liminf _{\varepsilon \rightarrow 0+} \frac{\mathcal{H}^{2}\left(A_{\varepsilon}\right)-\mathcal{H}^{2}(A)}{\varepsilon} .
$$

However, we shall refer to $\mu_{+}(A)$ as the Minkowski perimeter of $A$ instead of the outer Minkowski content of $\partial A$. 
Theorem 6.1. If $0<a<1$ and $A$ is an $\mathcal{H}^{2}$-measurable subset of $E(a)$, then

$$
\mathcal{H}^{2}(A) \leq \frac{a}{\sqrt{1-a^{2}}} P(A)
$$

with equality if and only if $A=E(a)$.

Proof. Since $A \subset E(a)$ we may, by replacing the spherical polygons $M_{i}$ in the definition of $P(A)$ by $M_{i} \cap E(a)$, if necessary, take these spherical polygons to be subsets of $E(a)$. From the definition of $P(A)$ it then follows that if (17) is false for $A$, there is a spherical polygon $M_{i}$ in $E(a)$ such that (17) is also false for $M_{i}$. Thus we may assume that $A$ itself is a spherical polygon with $\mathcal{H}^{2}(A)>0$. We may further assume that $\operatorname{int} A$ is connected, since if (17) is false, there must be a component of int $A$ such that (17) is also false when $A$ is replaced by the closure of this component.

Choose $0<b \leq a$ and $0<\gamma \leq 2 \pi$ to be minimal such that int $A \subset C=$ $E(b) \cap S(\gamma)$ for some sector $S(\gamma)$. Clearly, $\mathcal{H}^{2}(A) \leq \mathcal{H}^{2}(C)=2 b \gamma$. We claim that

$$
P(A) \geq 2 \gamma \sqrt{1-b^{2}},
$$

with equality if and only if $A=E(b)$. This will suffice to prove the theorem, since it then follows that

$$
\mathcal{H}^{2}(A) \leq 2 b \gamma \leq \frac{b}{\sqrt{1-b^{2}}} P(A) \leq \frac{a}{\sqrt{1-a^{2}}} P(A),
$$

with equality if and only if $A=E(a)$.

To prove the claim, assume first that $\gamma<2 \pi$. The set $C \cap \partial S(\gamma)$ consists of two arcs, $A_{1}$ and $A_{2}$ say, and $A$, being closed, must contain a point in each arc. Therefore $\partial A$ contains two polygonal arcs, that is, spherical polygonal arcs, disjoint except at their endpoints, each joining $A_{1}$ and $A_{2}$.

The shortest polygonal arcs in $C$ joining $A_{1}$ and $A_{2}$ are the two arcs that comprise $C \cap \partial E(b)$, each of which has length $\gamma \sqrt{1-b^{2}}$. To see this, consider an arbitrary polygonal arc $Q$ in $C$ joining $A_{1}$ and $A_{2}$. Let $Q=\left\{(x(t), y(t), z(t)) \in S^{2}: 0 \leq\right.$ $t \leq 1\}$, where $x(t)=\cos \theta(t) \sin \phi(t), y(t)=\sin \theta(t) \sin \phi(t)$, and $z(t)=\cos \phi(t)$, in spherical polar coordinates. Here $\theta(t)$ and $\phi(t)$ are continuous functions on $[0,1]$ and there exists a finite partition $0=t_{0}<t_{1}<\cdots<t_{N-1}<t_{N}=1$ such that both functions are continuously differentiable in $\left[t_{i}, t_{i+1}\right]$, for $i=0,1, \ldots, N-1$. Since $Q \subset E(b)$, we have $|\cos \phi(t)| \leq b$ for $0 \leq t \leq 1$. Therefore the length of $Q$ is

$$
\begin{aligned}
\sum_{i=0}^{N-1} \int_{t_{i}}^{t_{i+1}} \sqrt{x^{\prime}(t)^{2}+y^{\prime}(t)^{2}+z^{\prime}(t)^{2}} d t & =\sum_{i=0}^{N-1} \int_{t_{i}}^{t_{i+1}} \sqrt{\phi^{\prime}(t)^{2}+\sin \phi(t)^{2} \theta^{\prime}(t)^{2}} d t \\
& \geq \sum_{i=0}^{N-1} \int_{t_{i}}^{t_{i+1}}|\sin \phi(t)|\left|\theta^{\prime}(t)\right| d t \\
& \geq \sqrt{1-b^{2}}|\theta(1)-\theta(0)| .
\end{aligned}
$$

If equality holds in the first of the previous two inequalities, then $\phi^{\prime}(t)=0$ and hence $\phi(t)$ is constant for $0 \leq t \leq 1$. If equality also holds in the second of these inequalities, then $|\cos \phi(t)|=b$ and $\theta(t)$ is monotonic for $0 \leq t \leq 1$. It follows that $Q$ is one of the two arcs that comprise $C \cap \partial E(b)$. This proves (18) when $\gamma<2 \pi$.

The above argument shows that the shortest polygonal arc in $E(b)$ joining two meridians is contained in $\partial E(b)$. With this in hand, (18) and its equality conditions follow easily in the remaining case when $\gamma=2 \pi$. 
Corollary 6.2. If $0<a<1$ and $A$ is an $\mathcal{H}^{2}$-measurable subset of $E(a)$, then

$$
\mathcal{H}^{2}(A) \leq \frac{a}{\sqrt{1-a^{2}}} \mu_{+}(A),
$$

with equality if and only if $A=E(a)$.

Proof. The inequality and equality condition both follow from Theorem 6.1 and the general inequality $P(A) \leq \mu_{+}(A)$ (see [4, Theorem 14.2.1]).

\section{Equatorial Symmetrization}

We begin by applying the isoperimetric inequality obtained in the previous section to prove a property of $\eta_{K}$ in $\mathbb{R}^{3}$.

Theorem 7.1. If $K$ is an o-symmetric convex body in $\mathbb{R}^{3}$, then $\eta_{K}(\rho) / \rho$ is decreasing.

Proof. Let $F(\rho)=\eta_{K}(\rho) / \rho$ and assume to the contrary that there exist $\rho_{1}<\rho_{2}$ such that $F\left(\rho_{1}\right)<F\left(\rho_{2}\right)$. Let $M=\sup \left\{F(\rho): \rho_{1} \leq \rho \leq \rho_{2}\right\}$. Clearly $F$ inherits the properties of $\eta_{K}$ established in Theorem 4.2(i) and in particular $F$ is continuous on the left and upper semicontinuous. Therefore $M$ is a maximum and the set $I=\left\{s \in\left[\rho_{1}, \rho_{2}\right]: F(s)=M\right\}$ is closed. Let $\rho_{0}$ be the minimum number in this set. Then for $0<\varepsilon \leq \varepsilon_{0}=\rho_{0}-\rho_{1}$, we have

$$
F\left(\rho_{0}-\varepsilon\right)<F\left(\rho_{0}\right) .
$$

Now let $A=\left(1 / \rho_{0}\right) K \cap S^{2}$, so that $\mathcal{H}^{2}(A)=F\left(\rho_{0}\right) / \rho_{0}$. Since $K$ is $o$-symmetric, $r_{K}>0$ and $K$ is contained in a closed slab of width $2 r_{K}$, bounded by two common supporting hyperplanes to $K$ and $r_{K} B$. It follows that if we let $a=r_{K} / \rho_{0}$, then $A \subset E(a)$.

If $A=E(a)$, then (20) cannot hold. Indeed, in that case, we would have $F(\rho) \geq$ $4 \pi r_{K}$ for all $\rho \in\left[r_{K}, \rho_{0}\right]$ with equality for $\rho=\rho_{0}$. Therefore $A \neq E(a)$.

Suppose that the Minkowski perimeter $\mu_{+}(A)$ of $A$ is finite. By (19), there is a $c>0$ such that

$$
\mathcal{H}^{2}(A)=\frac{a}{\sqrt{1-a^{2}}}\left(\mu_{+}(A)-c\right) .
$$

By the definition of $\mu_{+}(A)$, there is an $\varepsilon_{1}>0$ such that

$$
\mathcal{H}^{2}\left(A_{\varepsilon}\right) \geq \mathcal{H}^{2}(A)+\varepsilon\left(\mu_{+}(A)-c / 2\right)
$$

for all $\varepsilon \in\left(0, \varepsilon_{1}\right)$.

Choose $\varepsilon>0$ so that $\varepsilon<\varepsilon_{0}$,

$$
\varepsilon<\frac{c \rho_{0}}{2 \mu_{+}(A)-c}
$$

and

$$
\phi=\arccos \frac{r_{K}}{\rho_{0}}-\arccos \frac{r_{K}}{\rho_{0}-\varepsilon}<\varepsilon_{1} .
$$

Using (20), (12), (22), and (24), we obtain

(25) $\frac{\rho_{0}}{\rho_{0}-\varepsilon} \mathcal{H}^{2}(A)=\frac{F\left(\rho_{0}\right)}{\rho_{0}-\varepsilon}>\frac{F\left(\rho_{0}-\varepsilon\right)}{\rho_{0}-\varepsilon} \geq \mathcal{H}^{2}\left(A_{\phi}\right) \geq \mathcal{H}^{2}(A)+\phi\left(\mu_{+}(A)-c / 2\right)$,

which gives

$$
\frac{\varepsilon}{\rho_{0}-\varepsilon} \mathcal{H}^{2}(A)>\left(\arccos \frac{r_{K}}{\rho_{0}}-\arccos \frac{r_{K}}{\rho_{0}-\varepsilon}\right)\left(\mu_{+}(A)-c / 2\right) .
$$


By (16), we have

$$
\arccos \frac{r_{K}}{\rho_{0}}-\arccos \frac{r_{K}}{\rho_{0}-\varepsilon}>\frac{\varepsilon r_{K}}{\rho_{0} \sqrt{\rho_{0}^{2}-r_{K}^{2}}}=\frac{\varepsilon a}{\rho_{0} \sqrt{1-a^{2}}} .
$$

Consequently

$$
\mathcal{H}^{2}(A)>\frac{\left(\rho_{0}-\varepsilon\right) a}{\rho_{0} \sqrt{1-a^{2}}}\left(\mu_{+}(A)-c / 2\right)
$$

This and (21) yield

$$
\mu_{+}(A)-c>\frac{\rho_{0}-\varepsilon}{\rho_{0}}\left(\mu_{+}(A)-c / 2\right),
$$

which is a contradiction to (23).

It remains to consider the case when $\mu_{+}(A)=\infty$. Using (20) but not the assumption that $\mu_{+}(A)$ is finite, the argument above leading to (25) yields instead the conclusion that for each $M \in \mathbb{N}$, there is an $0<\varepsilon<\varepsilon_{0}$ such that

$$
\frac{\rho_{0}}{\rho_{0}-\varepsilon} \mathcal{H}^{2}(A)=\frac{F\left(\rho_{0}\right)}{\rho_{0}-\varepsilon}>\frac{F\left(\rho_{0}-\varepsilon\right)}{\left(\rho_{0}-\varepsilon\right)} \geq \mathcal{H}^{2}\left(A_{\phi}\right) \geq \mathcal{H}^{2}(A)+\phi M .
$$

Now the argument above leading to (26) gives

$$
\mathcal{H}^{2}(A)>\frac{\left(\rho_{0}-\varepsilon\right) a}{\rho_{0} \sqrt{1-a^{2}}} M,
$$

a contradiction to $\mathcal{H}^{2}(A)<\mathcal{H}^{2}(E(a))$ if $M$ is sufficiently large.

Theorem 7.1 has the following nice interpretation. Let $K$ be a convex body containing the origin in $\mathbb{R}^{n}$. For each $\rho \in\left[r_{K}, R_{K}\right]$, choose $h(\rho)$ such that

$$
\mathcal{H}^{n-1}\left(\left\{x \in \mathbb{R}^{n}:-h(\rho) \leq x_{n} \leq h(\rho)\right\} \cap \rho S^{n-1}\right)=\eta_{K}(\rho),
$$

and let

$$
E_{K}=r_{K} B \cup \bigcup\left\{\left\{x \in \mathbb{R}^{n}:-h(\rho) \leq x_{n} \leq h(\rho)\right\} \cap \rho S^{n-1}: r_{K} \leq \rho \leq R_{K}\right\} .
$$

We call $E_{K}$ the equatorial symmetral of $K$. Clearly $E_{K}$ is a body of revolution about the $x_{n}$-axis. If $K$ is $o$-symmetric, it is not even obvious a priori that $E_{K}$ is a star body, but when $n=3$, Theorem 7.1 yields much more, namely, that $E_{K}$ is "quasiconvex" in the sense that the function $h$ is decreasing. Indeed, when $n=3$ we have

$$
\mathcal{H}^{2}\left(\left\{x \in \mathbb{R}^{3}:-h(\rho) \leq x_{3} \leq h(\rho)\right\} \cap \rho S^{2}\right)=4 \pi \rho h(\rho),
$$

and hence $h(\rho)=\eta_{K}(\rho) /(4 \pi \rho)$ is decreasing.

When $n=2$, the equatorial symmetral of $K$ coincides with the semicircular symmetral introduced by Bonnesen [2, p. 67]. This process is called annular symmetrization by Bonnesen and Fenchel [3, p. 77]. Bonnesen proved that if the center of the minimal annulus containing $\partial K$ is at the origin, then the perimeter of $E_{K}$ does not exceed that of $K$. The semicircular symmetral is used by Campi [5] to obtain stability estimates connected to the isoperimetric inequality.

Another form of symmetral, the spherical symmetral, is the natural generalization of the circular symmetral introduced by Pólya [17. and discussed at length by Pólya and Szegö [18, p. 193]. The spherical symmetral of $K$ is the body produced by replacing $K \cap \rho S^{n-1}$ by a cap in $\rho S^{n-1}$ of the same $\mathcal{H}^{n-1}$-measure, centered at the point on the positive $x_{1}$-axis a distance $\rho$ from the origin. The spherical symmetral of an $o$-symmetric convex body does not generally enjoy the corresponding 
quasiconvexity property, however; this is shown by straightforward computation when $K=\left\{-r_{K} \leq x_{n} \leq r_{K}\right\} \cap R B$ where $R>r_{K}$.

There seems to be no obvious direct relationship between Conjecture 4.6 and Theorem 7.1, Indeed, when $\eta_{K}^{\prime}(\rho)$ exists, Theorem 7.1 states that

$$
\eta_{K}^{\prime}(\rho) \leq \frac{\eta_{K}(\rho)}{\rho}
$$

Therefore when $\eta_{K}$ is differentiable, Conjecture 4.6 implies Theorem 7.1 if $\alpha_{K} \geq$ $\rho \eta_{K} / 2$ and Theorem 7.1] implies Conjecture 4.6] if $\alpha_{K} \leq \rho \eta_{K} / 2$, while we only know in general that $\alpha_{K} \geq \rho \eta_{K} / 3$.

\section{Related Functions}

There is another pair of functions that are related to those considered above and seem worthy of study. Let $K$ and $L$ be convex bodies in $\mathbb{R}^{n}$. Let

$$
m(K, L)=\max _{x \in \mathbb{R}^{n}} V(K \cap(L+x))
$$

and let

$$
s(K, L)=\max _{x \in \mathbb{R}^{n}} \mathcal{H}^{n-1}(K \cap(\partial L+x)) .
$$

We shall restrict our attention to the former function and define its kernel by

$$
\bar{K}_{L}=\left\{x \in \mathbb{R}^{n}: V(K \cap(L+x))=m(K, L)\right\} .
$$

Note that when $\rho>0$ is such that $\rho B+x \subset K$ for some $x \in \mathbb{R}^{n}, \bar{K}_{\rho B}$ is just an inner parallel body of $K$ (see [20, p. 134]).

For $r(K, L) \leq \rho \leq R(K, L)$, let

$$
\bar{\alpha}_{K}(L, \rho)=m(K, \rho L) .
$$

Note that when $K$ and $L$ are $o$-symmetric, $\bar{\alpha}_{K}(L, \rho)=\alpha_{K}(L, \rho)$.

It is worth remarking that the function $m(K, L)$ appears in a fascinating conjecture of Dar [6], that if $K$ and $L$ are convex bodies in $\mathbb{R}^{n}$, then

$$
V(K+L)^{1 / n} \geq m(K, L)^{1 / n}+\left(\frac{V(K) V(L)}{m(K, L)}\right)^{1 / n} .
$$

Dar shows that (28) implies the Brunn-Minkowski inequality for convex bodies. He proves (28) when $K$ and $L$ are ellipsoids and for some other special cases, but his conjecture seems to be open even for planar o-symmetric bodies.

Theorem 8.1. If $K$ and $L$ are convex bodies in $\mathbb{R}^{n}$, then $\bar{\alpha}_{K}(L, \rho)^{1 / n}$ is concave.

Proof. This is similar to the proof of Theorem 3.1. Let $0<\rho_{1}<\rho_{2}$ and let $z_{i} \in \bar{K}_{\rho_{i} L}, i=1,2$. Let $H=K \times\left[\rho_{1}, \rho_{2}\right] \subset \mathbb{R}^{n+1}$, for $i=1,2$ let

$$
D_{i}=\left\{\left(x_{1}, \ldots, x_{n+1}\right):\left(x_{1}, \ldots, x_{n}\right) \in \rho_{i} L+z_{i}, x_{n+1}=\rho_{i}\right\}
$$

and let $C=\operatorname{conv}\left\{D_{1}, D_{2}\right\}$. Finally, let $M=H \cap C$.

Let $\rho_{1}<\rho<\rho_{2}$ and choose $0<t<1$ such that $\rho=(1-t) \rho_{1}+t \rho_{2}$. Let $z=(1-t) z_{1}+t z_{2}$. Note that

$$
C \cap\left\{x_{n+1}=\rho\right\}=\left\{\left(x_{1}, \ldots, x_{n+1}\right):\left(x_{1}, \ldots, x_{n}\right) \in \rho L+z, x_{n+1}=\rho\right\},
$$

so identifying the plane $\left\{x_{n+1}=\rho\right\}$ with $\mathbb{R}^{n}$, we have

$$
M \cap\left\{x_{n+1}=\rho\right\}=K \cap(\rho L+z) .
$$


Similarly, identifying the plane $\left\{x_{n+1}=\rho_{i}\right\}$ with $\mathbb{R}^{n}$, we have

$$
M \cap\left\{x_{n+1}=\rho_{i}\right\}=H \cap D_{i}=K \cap\left(\rho_{i} L+z_{i}\right),
$$

for $i=1,2$. By the Brunn-Minkowski inequality, we obtain

$$
\begin{aligned}
\bar{\alpha}_{K}(L, \rho)^{1 / n} & \geq V(K \cap(\rho L+z))^{1 / n} \\
& \geq(1-t) V\left(K \cap\left(\rho_{1} L+z_{1}\right)\right)^{1 / n}+t V\left(K \cap\left(\rho_{2} L+z_{2}\right)\right)^{1 / n} \\
& =(1-t) \bar{\alpha}_{K}\left(L, \rho_{1}\right)^{1 / n}+t \bar{\alpha}_{K}\left(L, \rho_{2}\right)^{1 / n},
\end{aligned}
$$

as required.

In view of the fact that $\bar{\alpha}_{K}(L, \rho)=\alpha_{K}(L, \rho)$ and $\bar{\eta}_{K}(L, \rho)=\eta_{K}(L, \rho)$ when $K$ and $L$ are $o$-symmetric, several of our earlier results apply to the new functions under this restriction. Natural questions arise when $K$ or $L$ is not $o$-symmetric, but we leave these for a future study.

\section{REFERENCES}

[1] R. Benguria, M. Levitin, and L. Parnovski, Fourier transform, null variety, and Laplacian's eigenvalues, J. Funct. Anal. 257 (2009), 2088-2123. MR2548031

[2] T. Bonnesen, Les problèmes des isopérimètres et des isépiphanes, Gauthier-Villars, Paris, 1929.

[3] T. Bonnesen and W. Fenchel, Theory of Convex Bodies, BCS Associates, Moscow, Idaho, 1987. MR.920366 (88j:52001)

[4] Y. D. Burago and V. A. Zalgaller, Geometric Inequalities, Springer, Berlin, 1988. MR936419 (89b:52020)

[5] S. Campi, Isoperimetric deficit and convex plane sets of maximum translative discrepancy, Geom. Dedicata 43 (1992), 71-81. MR.1169365 (93d:52012)

[6] S. Dar, A Brunn-Minkowski-type inequality, Geom. Dedicata 77 (1999), 1-9. MR 1706512 (2000i:52021)

[7] R. J. Gardner, A positive answer to the Busemann-Petty problem in three dimensions, Ann. of Math. (2) 140 (1994), 435-447. MR1298719 (95i:52005)

[8] R. J. Gardner, The Brunn-Minkowski inequality, Bull. Amer. Math. Soc. 39 (2002), 355-405. MR.1898210 (2003f:26035)

[9] R. J. Gardner, Geometric Tomography, second edition, Cambridge University Press, New York, 2006. MR2251886 (2007i:52010)

[10] R. J. Gardner, A. Koldobsky, and T. Schlumprecht, An analytical solution to the BusemannPetty problem on sections of convex bodies, Ann. of Math. (2) 149 (1999), 691-703. MR.1689343 (2001b:52011)

[11] R. J. Gardner, E. B. Vedel Jensen, and A. Volčič, Geometric tomography and local stereology, Adv. in Appl. Math. 30 (2003), 397-423. MR.1973951 (2004e:28006)

[12] P. M. Gruber, Convex and Discrete Geometry, Springer, Berlin, 2007. MR.2335496 (2008f:52001)

[13] G. H. Hardy, J. E. Littlewood, and G. Pólya, Inequalities, Cambridge University Press, Cambridge, 1959.

[14] E. Lutwak, Dual mixed volumes, Pacific J. Math. 58 (1975), 531-538. MR0380631 (52:1528)

[15] E. Lutwak, Mean dual and harmonic cross-sectional measures, Ann. Mat. Pura Appl. (4) 119 (1979), 139-148. MR551220 (80m:52007)

[16] E. Lutwak, Intersection bodies and dual mixed volumes, Adv. Math. 71 (1988), 232-261. MR.963487 (90a:52023)

[17] G. Pólya, Sur la symétrisation circulaire, C. R. Acad. Sci. Paris 230 (1950), 25-27. MR0033402 (11:435h)

[18] G. Pólya and G. Szegö, Isoperimetric Inequalities in Mathematical Physics, Princeton University Press, Princeton, 1951. MR0043486 (13:270d)

[19] J. R. Sangwine-Yager, Bonnesen-style inequalities for Minkowski relative geometry, Trans. Amer. Math. Soc. 307 (1988), 373-382. MR 936821 (89g:52007) 
[20] R. Schneider, Convex Bodies: The Brunn-Minkowski Theory, Cambridge University Press, Cambridge, 1993. MR1216521 (94d:52007)

[21] Gaoyong Zhang, Intersection bodies and the Busemann-Petty inequalities in $\mathbb{R}^{4}$, Ann. of Math. (2) 140 (1994), 331-346. MR.1298716 (95i:52004)

[22] Gaoyong Zhang, A positive answer to the Busemann-Petty problem in four dimensions, Ann. of Math. (2) 149 (1999), 535-543. MR1689339(2001b:52010)

Dipartimento di Ingegneria dell'Informazione, Università degli Studi di Siena, Via Roma 56, 53100 Siena, Italy

E-mail address: campi@dii.unisi.it

Department of Mathematics, Western Washington University, Bellingham, WashingTON 98225-9063

E-mail address: Richard.Gardner@wwu.edu

Dipartimento di Matematica e Applicazioni per l'Architettura, Università degli Studi di Firenze, Piazza Ghiberti 27, 50122 Firenze, Italy

E-mail address: paolo@fi.iac.cnr.it 